EPJ Web of Conferences 77, 00022 (2014)

DOI: $10.1051 /$ epjconf $/ 20147700022$

(C) Owned by the authors, published by EDP Sciences, 2014

\title{
Metrological Issues in Molecular Radiotherapy
}

\author{
Marco D’Arienzo ${ }^{1,2}$, Marco Capogni ${ }^{1}$, Vere Smyth $^{3}$, Maurice Cox ${ }^{3}$, Lena Johansson ${ }^{3}$, Jaroslav Solc ${ }^{4}$, Christophe Bobin 5 , \\ Hans Rabus ${ }^{6}$, Leila Joulaeizadeh ${ }^{7}$ \\ ${ }^{1}$ National Institute of Ionizing Radiation Metrology, ENEA-INMRI, C.R. Casaccia, 00123 Rome, Italy \\ 2 Department of Human Anatomy, Histology, Forensic Medicine and Orthopedics, Sapienza University of Rome, Via \\ Borelli 50, 00161 Rome, Italy \\ ${ }^{3}$ National Physical Laboratory NPL, Hampton Road, Teddington, Middlesex, TW11 0LW, UK \\ ${ }^{4}$ Czech Metrology Institute (CMI), Inspectorate for Ionising Radiation, Radiova 1, CZ-102 00 Prague 10, Czech Republic \\ ${ }^{5}$ Commissariat a l'Energie Atomique (CEA) Bt 476, Pt Courrier 142, CEA-Saclay, FR-91191 Gif-sur-Yvette Cedex, France \\ ${ }^{6}$ Physikalisch-Technische Bundesanstalt (PTB), Bundesallee 100, D-38116 Braunschweig, Germany \\ ${ }^{7}$ VSL, Dutch Metrology Institute, Thijsseweg 11, P.O. Box 654, NL-2629, JA Delft, Netherlands
}

\begin{abstract}
The therapeutic effect from molecular radiation therapy (MRT), on both tumour and normal tissue, is determined by the radiation absorbed dose. Recent research indicates that as a consequence of biological variation across patients the absorbed dose can vary, for the same administered activity, by as much as two orders of magnitude. The international collaborative EURAMET-EMRP project "Metrology for molecular radiotherapy (MetroMRT)" is addressing this problem. The overall aim of the project is to develop methods of calibrating and verifying clinical dosimetry in MRT. In the present paper an overview of the metrological issues in molecular radiotherapy is provided.
\end{abstract}

\section{Introducing the problem of molecular radiation therapy}

Molecular radiation therapy (MRT), also known as targeted radionuclide therapy, is a unique form of radiotherapy where a high radiation dose is delivered internally, ideally to a specific target. The therapeutic agent may be administrated in several ways: ingestion, intravenous infusion, injection to a body cavity or pathological space (locoregional therapy) or direct injection into a solid tumour.

Molecular radiotherapy is routinely prescribed on the basis of administered activity of the therapeutic agent. However, uptake and retention differ from patient to patient and therefore the individual dose to the target can vary between patients given the same administered activity. Recent research indicates the range of variation can be up to two orders of magnitude, which is particularly alarming from the point of view of radiation protection $[1,2]$. At the low extreme, the patient might gain negligible therapeutic benefit. At the high extreme, the patient might receive more radiation than is needed to treat the tumour. Both cases technically constitute maladministrations.

Recent research and new technology developments have permitted estimates of radiation dose in individual patients to target tissues and critical normal tissues at risk to be obtained. However, there has been almost no adoption of these methodologies in routine clinical MRT practice. The reasons are many, but, mainly because the methodology is difficult, there is no standardization of procedures, and there is no objective means of predicting the effect of individual patient dosimetry on treatment outcomes.

In fact, when compared with conventional external beam radiotherapy, in which individual patient dosimetry is mandatory and strictly controlled according to agreed protocols, there is full traceability to primary standards, and there are even legal requirements for accuracy (within $5 \%$ of a reference value), it is clear that MRT is urgently in need of metrological support in order to bring dosimetry practice to an acceptable and comparable standard.

The new international collaborative EURAMETEMRP project "Metrology for molecular radiotherapy" (MetroMRT) is addressing this problem. The project is developing the background metrology to support routine individual MRT patient dosimetry, and together with a programme of dissemination is working with the clinical community to achieve widespread implementation. The outcomes from this project will enable personalized treatment planning, based on individualized patient

\footnotetext{
${ }^{\text {a }}$ Corresponding author: caroline.le.bihan@ifremer.fr
} 
dosimetry, which will have a direct and potentially significant benefit.

The project formulates MRT dosimetry as a measurement chain that is traceable to primary standards with given uncertainty (analogous to external beam radiotherapy). The links in the chain are I) measurement of administered activity; II) sequence of activity measurements within a defined tissue volume in the patient through quantitative imaging (QI) procedures; III) construction of an activity-time function from the sequence; IV) integration of the activity-time function; V) calculation of the absorbed dose from the activity-time integral.

\section{Measurement of administered activity}

As a general rule, the metrology of radionuclide activity is a mature science. However the radionuclides used for therapy necessarily have a significant proportion of (or in some cases only) short-range emissions (beta particles, Auger electrons, alpha particles) in order to deliver a local absorbed dose. This fact requires dedicated methods for accurate activity measurement, both at the primary standard level and in the clinic. As an example, ${ }^{90} \mathrm{Y}$ microspheres are an important therapeutic option used in the treatment of liver cancer through a process known as selective internal radiation therapy (SIRT). SIRT is performed by injecting ${ }^{90} \mathrm{Y}$ resin or glass microspheres directly into the hepatic artery - the blood supply of liver lesions is mainly derived from this artery, whereas normal liver tissue receives about $80 \%$ of its blood supply from the portal vein. By infusing the microspheres into the hepatic artery, a high radiation dose can be delivered selectively into the tumour while sparing normal liver tissue that surrounds the tumour compartments.

Beta emitters on microspheres pose a particular problem for activity measurement because of radiation absorption by the microspheres. Moreover, there is presently no traceability to national and international standards for these microspheres.

At present there are two available microsphere types, resin and glass. Resin microsphere (SIR-spheres ${ }^{\circledR}$ ) are provided in a vial containing approximately $3 \mathrm{GBq}$ of activity in a $5 \mathrm{~mL}$ solution of sterile water, while glass microspheres (TheraSphere ${ }^{\circledR}$ ) are supplied in $0.6 \mathrm{ml}$ of sterile, pyrogen-free water contained in a $1.0 \mathrm{ml} \mathrm{v}$-bottom vial secured within a $12 \mathrm{~mm}$ clear acrylic vial shield.

Previous work by Mo et al. [3] described the development of an activity standard for resin microspheres. In their work the authors first performed primary and secondary standardizations on a ${ }^{90} \mathrm{Y}$ solution. Then, through chemical digestion of the resin microspheres, a secondary standard factor for the ${ }^{90} \mathrm{Y}$ solution was converted into a secondary standard factor for the microspheres. Finally, ARI (Australian Radiopharmaceuticals and Industrials) production chamber and ARI QC TPA and Vinten chambers were calibrated for the $1 \mathrm{ml}$ and $5 \mathrm{ml}$ microsphere samples in Wheaton vials, where the calibration factor for $5 \mathrm{ml}$ may be derived from the factor for $1 \mathrm{ml}$ microspheres (although introducing a relatively large uncertainty).

Thus, evidently there is a need to establish a capability for accurately measuring the activity of ${ }^{90} \mathrm{Y}$ microspheres to traceable measurement standards. In fact, one of the tasks of the MetroMRT project is to develop the Triple to Double Coincidence Ratio (TDCR) method using Cherenkov emission for the purpose of improving the accuracy of administered activity to patients for such highenergy beta-emitters (e.g. ${ }^{90} \mathrm{Y},{ }^{89} \mathrm{Sr}$ and ${ }^{32} \mathrm{P}$ ).

Developed for radionuclide standardization using liquid scintillation, the TDCR method is based on a specifically designed 3-photomultipliers system [4]. Knowing the radionuclide decay scheme, the activity is determined using a free-parameter statistical model that allows a mathematical relationship between the detector efficiency (double coincidences between photomultipliers) and the experimental TDCR ratio given by coincidences between photomultipliers to be established. The TDCR value is interpreted as an indicator of the detection efficiency depending on the radionuclide to be standardized.

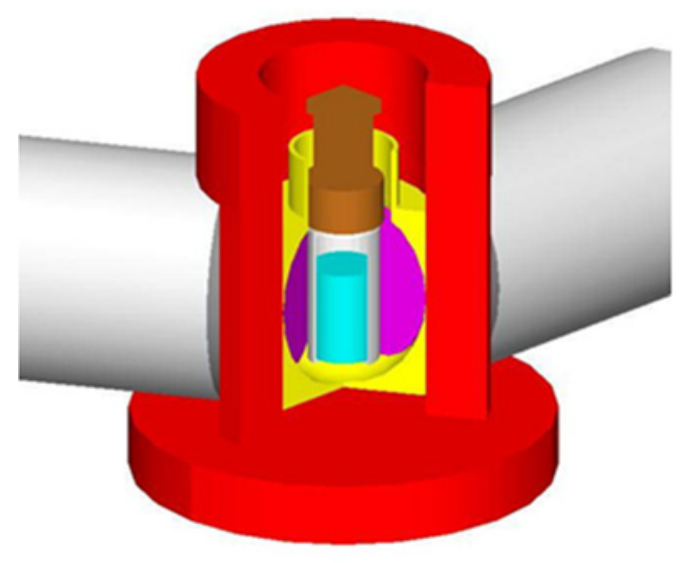

Figure 1: Geometry modeling of the TDCR counter with the Geant4 code

Using the same detection set-up, the TDCR method can be extended to Cherenkov emission. In this case, light emission is characterized by a threshold effect that limits the production of photons by a charged particle (Cherenkov photons result from an electromagnetic perturbation in a transparent medium such as aqueous solutions) and permits discriminating low-energy radioactive impurities in the measurements, usually performed in classical Liquid Scintillation Counting (LSC) techniques by taking into account the different half-life of each impurity of the solution [5]. Nevertheless, Cherenkov measurements are not frequently used for radionuclide standardization because higher detection efficiencies are obtained with liquid scintillation. However, the application of the TDCR method using Cherenkov emission is an interesting alternative to liquid scintillation in the case of high-energy beta-emitters used as radiopharmaceuticals for molecular radiotherapy, as ${ }^{90} \mathrm{Y}$ resin or glass microspheres. Indeed, since measurements are directly carried out in aqueous 
solutions, source preparation of radionuclides is easier and mixing with a liquid scintillator is avoided. The method should also minimize the influence of possible gammaemitting impurities in the activity measurements. For activity calculation, the modelling related to TDCRCherenkov measurements has to be modified in order to take into account the physical characteristics of Cherenkov effect (anisotropy of light emission, threshold effect, continuous spectral bandwidth). The implementation of the new modelling can be carried out according to two approaches. The classical analytical model can be constructed using a free parameter for the anisotropy of light emission [6]. By using the Monte Carlo code Geant4 [7], the other approach is based on a comprehensive description of the geometry and optical properties of the detector (see figure 1). In that stochastic modelling, triple and double coincidences between photomultipliers are calculated from the simulation of Cherenkov photons created in the optical cavity to the production of photoelectrons in photomultipliers [8].

Finally, a transfer protocol for end-users in the clinic is being developed, with the aim of improving their measurement techniques in order to reduce the standard uncertainty for activity delivery from $20 \%$ to less than 10 $\%$.

\section{Sequence of activity measurements within a defined tissue volume in the patient through quantitative imaging procedures}

Accurate determination of the activity of a radionuclide within a defined anatomy-indexed volume in a patient is fundamental to estimation of the absorbed dose to a critical target.

The measurement of radioactivity within a patient requires quantitative imaging procedures, for which there is currently no agreed measurement chain that gives traceability to a radioactivity standard.

The current state of the art for quantitative activity measurement is the use of combined SPECT-CT or PETCT imaging to give a 3D distribution of activity within the patient. Currently, SPECT-based quantitative imaging is more widely used than PET, as most therapeutic radionuclides emit photons suitable only for SPECT (Table 1). Recently, the possibility of performing PET quantitative imaging with ${ }^{90} \mathrm{Y}$ exploiting the extremely small $\beta^{+}$branch ratio of this radionuclide has been proposed by several authors and applied both to SIRT treatments [9-14] and locoregional therapies that use ${ }^{90} \mathrm{Y}$ as therapeutic agent [15].

To give absolute activity, the system must be calibrated and each image must be corrected for scatter, attenuation, partial volume effect, and dead time. While considerable work has been done in the application of correction techniques to both the filtered back-projection and iterative image reconstruction methodologies [16], there is no validated standard protocol, or any established methods for calibration or verification of system performance.

Furthermore, with the advent of hybrid SPECT-CT and PET-CT, more accurate localization of the volume of interest is possible. Since the imaging equipment has not been optimized for quantitative measurement, there are metrological issues that must be addressed.

MetroMRT is investigating the development of a procedure to calibrate a quantitative image measurement (using SPECT, PET, planar imaging etc.) in such a way that the calibration remains valid (within a given uncertainty) when the same imaging equipment is used on a patient.

Feasibility trials are being carried out with commonly used phantoms, such as the Jaszczak phantom, containing an accurately measured activity (or activities) traceable to a standard activity.

An anthropomorphic phantom will be used in a similar way to test the accuracy of quantitative imaging procedures over a range of clinically realistic geometries, given verified accuracy on a standard calibration phantom.

\begin{tabular}{|c|c|c|c|c|}
\hline Radionuclide & $\mathbf{T}_{1 / 2}$ & Decay & $\begin{array}{c}\mathbf{E}_{\beta}(\max ) \\
(\mathrm{MeV})\end{array}$ & $\begin{array}{c}\mathbf{E}_{\gamma} \\
(\mathbf{k e V})\end{array}$ \\
\hline$\overline{{ }^{32} \mathbf{P}}$ & $14.3 \mathrm{~d}$ & $\overline{\beta^{-}}$ & 1.70 & None \\
\hline${ }^{64} \mathrm{Cu}$ & $12.7 \mathrm{~h}$ & $\begin{array}{c}\beta^{-}, \mathrm{EC} \\
\beta^{+}\end{array}$ & $\begin{array}{l}\beta^{-}=0.58 \\
\beta^{+}=0.65\end{array}$ & None \\
\hline${ }^{67} \mathrm{Cu}$ & $2.58 \mathrm{~d}$ & $\beta^{-}$ & 0.58 & $\begin{array}{c}91(7 \%) \\
93(16 \%) \\
185(49 \%)\end{array}$ \\
\hline${ }^{67} \mathbf{G a}$ & $3.26 \mathrm{~d}$ & $\mathrm{EC}$ & & $\begin{array}{c}91(7 \%) \\
93(16 \%) \\
185(49 \%) \\
300(17 \%) \\
394(5 \%)\end{array}$ \\
\hline${ }^{89} \mathrm{Sr}$ & $50.5 \mathrm{~d}$ & $\beta^{-}$ & 1.49 & None \\
\hline${ }^{90} \mathrm{Y}$ & $2.67 \mathrm{~d}$ & $\beta^{-}$ & 2.28 & None \\
\hline${ }^{111} \mathbf{I n}$ & $2.8 \mathrm{~d}$ & EC & & $\begin{array}{l}171(90 \%) \\
245(94 \%)\end{array}$ \\
\hline${ }^{117 \mathrm{~m}} \mathrm{Sn}$ & $13.6 \mathrm{~d}$ & IT & & $159(86 \%)$ \\
\hline${ }^{131} \mathbf{I}$ & $8.02 \mathrm{~d}$ & $\beta^{-}$ & 0.61 & $\begin{array}{c}80(2.6 \%) \\
284(6 \%) \\
364(82 \%) \\
637(7 \%) \\
723(1.8 \%)\end{array}$ \\
\hline${ }^{153} \mathrm{Sm}$ & $1.95 \mathrm{~d}$ & $\beta^{-}$ & 0.81 & $103(30 \%)$ \\
\hline${ }^{166} \mathrm{Ho}$ & $26.8 \mathrm{~h}$ & $\beta^{-}$ & 1.85 & $\begin{array}{c}81(7 \%) \\
1,379(0.93 \%) \\
1,582(0.19 \%) \\
1,662(0.12 \%)\end{array}$ \\
\hline${ }^{177} \mathbf{L u}$ & $6.71 \mathrm{~d}$ & $\beta^{-}$ & 0.50 & $\begin{array}{c}113(6 \%) \\
208(11 \%)\end{array}$ \\
\hline${ }^{186} \mathrm{Re}$ & $3.72 \mathrm{~d}$ & $\mathrm{EC}, \beta^{-}$ & 1.07 & $137(9 \%)$ \\
\hline${ }^{188} \mathrm{Re}$ & $17.0 \mathrm{~h}$ & $\beta^{-}$ & 2.12 & $\begin{array}{c}155(15 \%) \\
478(1 \%) \\
633(1 \%)\end{array}$ \\
\hline
\end{tabular}

Table 1. Physical properties of selected radionuclides used either for therapy or imaging purposes in MRT applications. EC = electron capture, IT $=$ internal transition, $\beta^{-/+}=$beta minus/plus decay

\section{Construction of an activity-time function}

The QI activity measurement points acquired referenced to the same volume and obtained in different time periods after uptake - are typically three to six in 
number (figure 2). A model function is selected and fitted to these points to interpolate between and extrapolate beyond them. There is no generally accepted form for this function in terms of the number of exponential terms. Moreover, depending on the choice made, this function represents a purely decaying process (figure 3) or the biokinetics of uptake and retention of the radiopharmaceutical [24-27]. Appropriate statistical tests can be made to inform the selection process [25].

It is important that in fitting the selected model function, account is taken of measurement uncertainties associated with the activity values and of any measurement covariances involved. Covariances can arise due to systematic effects that influence all QI activity measurement points or as a result of the image reconstruction process. Some published approaches form a weighted fit based on the assumption of constant uncertainties or uncertainties that are proportional to activity values. In the current work, uncertainties are based on knowledge of the quantitative images used.

\section{Integration of the time sequence of activity measurements and calculation of absorbed dose}

The cumulative activity $\tilde{A}$ is estimated by integrating from time zero to infinity the function fitted to the activitytime points.
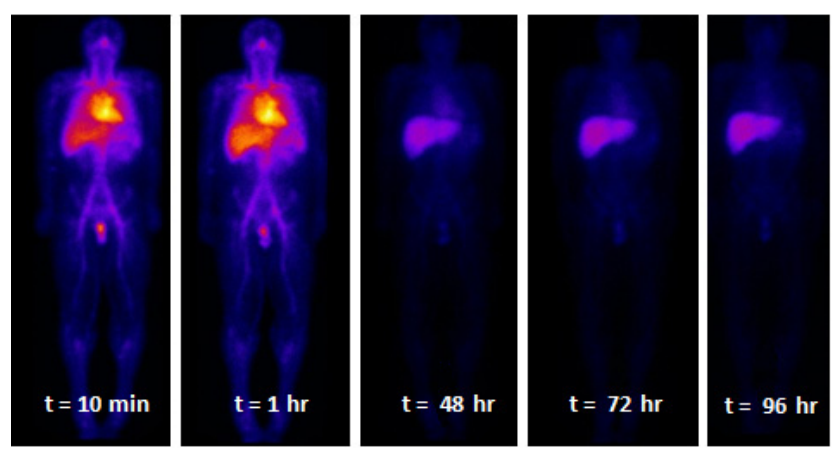

Figure 2. Biokinetics at different time points of ${ }^{111} \mathrm{In}, 185 \mathrm{MBq}$, intravenously administered.

Conceptually, absorbed dose determination requires the spatial assessment of radioactivity biodistribution (spatial analysis) in addition to cumulated activity assessment from the limited number of QI activity measurement points (time analysis), i.e., the total number of nuclear disintegrations in the volume of interest. Mathematically, the absorbed dose $D$ to a target volume is

$$
D=\tilde{A} \cdot S,
$$

where $S$ is the absorbed dose in the target region per nuclear transformation in the source region. An estimate of $\tilde{A}$ requires traceability to a standard of radionuclide activity, whilst an estimate of $S$ requires a standard of absorbed dose.

There are various ways of obtaining $S$-values (MIRD, RADAR, Monte Carlo calculation, etc.), but each is based on calculations from radionuclide spectral data rather than measurements [17, 18].

Hence, it is clear that calculating the absorbed dose from a radionuclide inside the body is complex, involving both the physics of radioactive decay and the biology of the body's metabolism.

Clinical dosimetry from radionuclides can be performed prior to, or after, the treatment. Depending on the approach taken, the calculation may use the standard MIRD formalism and $S$-factors (either organ-averaged or voxel-based) or an individualised calculation using Monte Carlo calculation. Whichever method is used, the result relies heavily on the accuracy of the nuclear data and the calculation techniques. Moreover, at present no general clinically accepted dosimetry protocol exists and dosimetry studies are usually performed on individual initiatives. As a consequence, dosimetry studies are rarely comparable. Traceable methods for the experimental verification of dose calculations need to be developed.

Previous work has shown differences between calculated and measured doses of some $10 \%$ to $30 \%$ for measurements in phantoms and for different radionuclides, while the standard uncertainty associated with patient dose delivery has been reported to be from $10 \%$ to $100 \%[1,2$, 19-23].

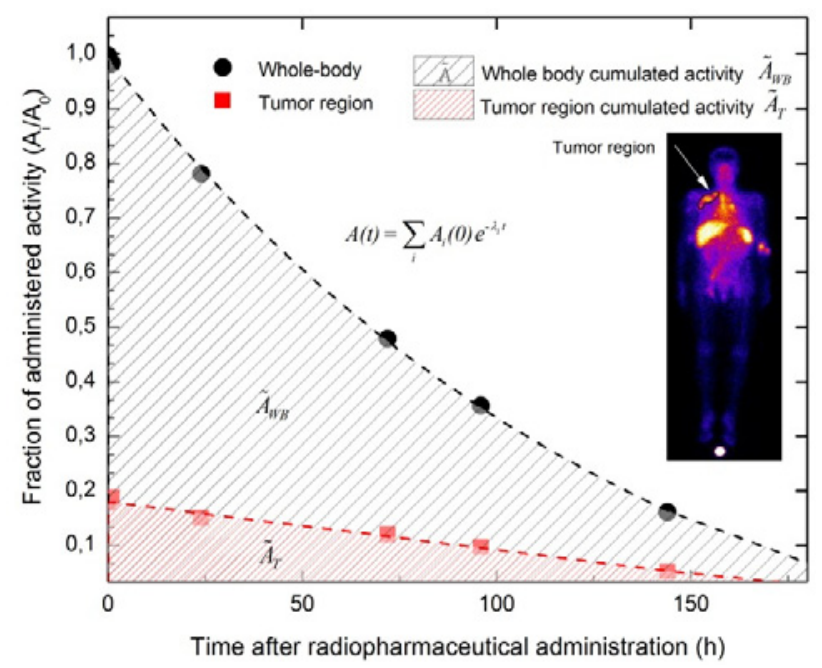

Figure 3. Integration of the time sequence of activity measurements. The cumulated activity $\tilde{A}$ needed for radiation dose calculations is determined by either graphical methods or multi-exponential curvefitting methods. Whole body and tumour region cumulated activities are reported.

MetroMRT is breaking new ground by developing new primary absorbed dose standards in order to be able to measure the dose from a radionuclide in solution, so that the calculations can be independently verified by measurement techniques calibrated against a standard. This work will lead to evaluation of the uncertainty associated with the usual methods. 
The first part of the work has involved a feasibility study of different technologies for possible use as standards. Methods considered include ionometry (extrapolation chamber), calorimetry (water and graphite calorimeter), and chemical dosimetry (dichromate solution).

\section{Modelling and uncertainty analysis}

Each link in the dosimetry chain is defined mathematically or represented by a computational model such as an iterative image reconstruction process or the determination of a specific activity-time function, The modelling also accounts for typical MRT treatment protocols. Each link in the chain constitutes a relationship between input quantities and output quantities, the latter becoming input quantities in the next link.

For each quantity an estimate is given by measurement or determined from a mathematical or computational model. Associated with each such estimate is a standard uncertainty (equivalent to a standard deviation). A standard uncertainty associated with an estimate of an output quantity for a link in the chain is given by propagating the standard uncertainties associated with the estimates of the input quantities through that link. There will be measurement covariances associated with estimates of several quantities when derived from common information, which have to be taken into account.

The mathematical model or the computational process used within a link may be a further source of uncertainty to be taken into account. For instance, an image reconstruction algorithm is typically not run to completion, that is, the process has not mathematically converged, because of the adverse effect of noise on the solution obtained. Instead, it is often run for a fixed number of iterations, which may be set by the supplier. The influence of this number is to be estimated. Another instance is the choice of biokinetic model for the activity-time function there are various forms of this model ranging from the number of exponential terms used to the precise form of the exponentials used (section 4). Different results will be obtained according to this choice and assessing the dispersion of results across a set of feasible models can be regarded as the contribution from "model uncertainty".

Overall, there is component uncertainty related to each link in the chain that when combined as above produce a dose estimate uncertainty (section 7). These component uncertainties constitute an uncertainty budget, analysis of which can be extremely informative in studying which aspects of the dosimetry chain could benefit from improvement (section 8).

In this work, internationally accepted principles [28-30] are applied for uncertainty propagation. References [29, 30] also apply when uncertainty propagation based on linearizing the model is not considered appropriate or when this form of uncertainty propagation is to be checked by propagating probability distributions rather than uncertainties.

\section{Evaluation of dose estimate uncertainty}

Because of the lack of validation against measurements aforementioned, there is no clear understanding or quantification of the uncertainty associated with an MRT dose estimate. For clinical reasons an overall standard uncertainty of around $10 \%$ is desirable, but at present it is not possible to determine its value. If this uncertainty were known, and in particular the component uncertainties it comprises that arise from the various links in the dosimetry chain, it would help to identify key elements of the dosimetry chain needing development. It would also provide a solid basis for obtaining an improvement in the quality of clinical dosimetry in MRT, for the benefit of patient care and clinical research. Furthermore, for the process of dose measurement to have legitimacy in terms of compliance with regulatory accuracy requirements, or for a component in the process, such as a software package, to achieve regulatory approval, it is essential that there is a formal procedure for obtaining the uncertainty budget for the measurement.

These issues are addressed in this work, especially in section 6 concerned with modelling and uncertainty analysis. In particular, the model, $D=\tilde{A} S$ [expression (1)], for the final link in the chain is very simple. The standard uncertainties associated with estimates of $\tilde{A}$ and $S$ are propagating through this expression to provide the standard uncertainty associated with the estimate of $D$. Earlier links in the chain will provide the "input uncertainties" to be used. These uncertainties are the difficult ones to establish as emphasized earlier.

\section{Conclusions}

A number of metrological issues in MRT still have to be addressed. The MetroMRT project will create an impact on the MRT community resulting from a) greater accuracy of the activity measurement of high-energy beta emitters in MRT clinics through development of new standards and protocols; b) greater accuracy in the determination of radiopharmaceutical activity uptake and retention in patients using QI techniques as a result of calibration and audit procedures, and recommendations for good practice; c) greater accuracy of determination of absorbed dose from cumulative activity of radiopharmaceuticals as a result of new verification methods and recommendations for good practice; d) objective knowledge of the uncertainties associated with alternative methods of MRT dosimetry allowing decisions to be made in MRT clinics on treatment protocols that will optimize patient benefits within available resources.

The overall impact that the MetroMRT project will have on the MRT community will be that, by regarding dosimetry as a formal traceable measurement with an associated uncertainty, the culture of treating patients with a nominal radiopharmaceutical activity will change to individualized patient treatments based on absorbed dose.

Using statistical data it will be possible to assess the implications for the health and medical research communities of reducing (or choosing not to reduce) individual uncertainties. One instance where uncertainty 
reduction is feasible is in the image reconstruction stage (section 6), where some form of regularization to prevent noise build-up can be used [31].

Generally, providing more accurate and hence more reliable results will be more expensive and must be balanced against foreseen benefits. The methodology developed here will provide an objective basis for making optimization decisions as to which parts of the uncertainty budget to address.

MetroMRT is analyzing the uncertainty implications in each of the methods used by the clinical research centres collaborating with the project, and will make recommendations on preferred protocols.

MRT will be much more accurately targeted, and as a direct result it will become more effective. With support from the metrology sector and with increasing confidence in the effectiveness of MRT, the modality will become much more widely used as part of the armamentarium against cancer.

\section{Acknowledgments}

The research leading to these results has received funding from the European Commission (EC) according to the EC Grant Agreement No 217257 between the EC and EURAMET e.V. (European Association of National Metrology Institutes) under the Seventh Framework Programme.

\section{References}

1. G.D. Flux, M. Bardiès, C. Chiesa et al, "Clinical radionuclide therapy dosimetry: the quest for the "Holy Gray"', Eur J Nucl Med Mol Imaging 34,1 (2007).

2. G.D. Flux, H. Masud, S.J. Chittenden et al, "A doseeffect correlation for radioiodine ablation in differentiated thyroid cancer", Eur J Nucl Med Mol Imaging 37:270-275 (2010).

3. L. Mo, B. Avci B, D. James, et al., "Development of activity standard for ${ }^{90} \mathrm{Y}$ microspheres". Appl. Radiat. Isot. 63, 193-199 (2005).

4. R. Broda, P. Cassette, K. Kossert, "Radionuclide metrology using liquid scintillation counting". Metrologia 44, S36-S52 (2007).

5. Capogni, M., De Felice P., Fazio, A., Latini F., Abbas K., 2008. "Development of a primary standard for calibration of ${ }^{64} \mathrm{Cu}$ activity measurement systems". Appl. Radiat. Isot. 66(6-7), 948-53 (2008).
6. K. Kossert, "Activity standardization by means of a new Čerenkov counting technique“. Appl. Radiat. Isot. 68, 1116-1120 (2010).

7. S. Agostinelli; et al., "Geant4 - a simulation toolkit". Nucl. Instrum. Meth. A 506, 250-303 (2003).

8. C. Bobin, C. Thiam, J. Bouchard, F. Jaubert, “ Application of stochastic TDCR model based on Geant4 for Cherenkov primary measurements", Appl. Radiat. Isot. 68, 2366-2371 (2010).

9. R. Lhommel, P. Goffette, M. Van den Eynde, et al "Yttrium-90 TOF PET scan demonstrates high-resolution biodistribution after liver SIRT". Eur J Nucl Med Mol Imaging 36, 1696 (2009)

10. L. Van Elmbt, S. Vandenberghe, S. Walrand, et al "Comparison of yttrium-90 quantitative imaging by TOF and non-TOF PET in a phantom of liver selective internal radiotherapy". Phys Med Biol 56:6759-6777 (2011)

11. S. Walrand, G.D. Flux, M.W. Konijnenberg, et al. "Dosimetry of yttrium-labelled radiopharmaceuticals for internal therapy: 86 Y or $90 Y$ imaging?" Eur J Nucl Med Mol Imaging 38 (suppl):S57-S68 (2011)

12. M. D'Arienzo, P. Chiaramida, L. Chiacchiararelli, et al:

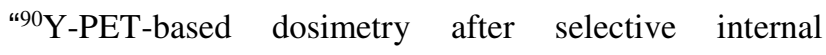
radiotherapy treatments". Nucl Med Commun 33:633-640 (2012)

13. O. Bagni, M. D'Arienzo, P. Chiaramida, L. Chiacchiararelli, et al "90Y-PET for the assessment of microsphere biodistribution after selective internal radiotherapy” Nucl Med Commun. 33(2):198-204 (2012).

14. K. Willowson, N. Forwood, B.W. Jakoby et al "Quantitative ${ }^{90} \mathrm{Y}$ image reconstruction in PET" Med Phys, 39:7153-7159 (2012).

15. C. Fabbri, V. Mattone, M. Casi et al "Quantitative evaluation on [90Y] DOTATOC PET and SPECT imaging by phantom acquisitions and clinical applications in locoregional and systemic treatments" QJNM 56:522-528 (2012)

16. V.V.A.A, Mathematical Techniques in Nuclear Medicine, Institute of Physics and Engineering in Medicine, (2011). 
17. M. Pacilio M, N. Lanconelli, S. Lo Meo et al, "Differences among Monte Carlo codes in the calculations of voxel $\mathrm{S}$ values for radionuclide targeted therapy and analysis of their impact on absorbed dose evaluations" Med Phys 36,1543 (2009).

18. J.M. Franquiz, S. Chigurupati, K. Kandagatla, "Beta voxel S values for internal emitter dosimetry" Med Phys 30,1030 (2003).

19. J.M. Pereira, M.J. Stabin, F.R. Lima et al, "Image Quantification for Radiation Dose Calculations Limitation and Uncertainties", Health Phys 99:688-701 (2012).

20. K. Sjogreen, M. Ljungberg, S.E. Strand, "An Activity Quantification Method Based on Registration of CT and Whole Body Scintillation Camera Images, with Application to ${ }^{131}$ I", J. Nucl. Med 43:972-982 (2002).

21. Y.K. Dewaraja, S.J. Wilderman, M. Ljungberg et al, "Accurate dosimetry in I-131 radionuclide therapy using patient specific 3-dimensional methods for SPECT reconstruction and absorbed dose calculation", J. Nucl. Med 46:840-849 (2005).

22. M. D’Arienzo, F. Cicone, L. Chiacchiararelli, “ThreeDimensional Patient-Specific Dosimetry in Radioimmunotherapy with ${ }^{90}$ Y-Ibritumomab-Tiuxetan", Cancer Biother Radiopharm 27:124-133 (2012).

23. M.G. Stabin, "Uncertainties in Internal Dose Calculations for Radiopharmaceutical", J. Nucl. Med, 49:853-860 (2008a).

24. M.G. Stabin, R.B. Sparks, E. Crowe, "OLINDA/EXM: The second-generation personal computer software for internal dose assessment in nuclear medicine", J. Nucl. Med, 46:1023-1027 (2005).

25. P. Kletting, S. Schimmel, H.A. Kestler, H. Hänscheid, M. Luster, "Molecular radiotherapy: The NUKFIT software for calculating the time-integrated activity coefficient", Medical Physics (to appear).

26. M.G. Cox, "Modelling clinical decay data using exponential functions". In Approximation algorithms for complex systems, A.I.E.H. Georgoulis and J. Levesley, Eds., Springer-Verlag, 184-203 (2011).
27. M.G. Cox, H.E. Joyce, J.C. Mason, “Approximation by sums of exponentials to decay functions using piecewise linear models". In Approximation theory IX, Volume I: Theoretical Analysis. C.K. Chui and L.L. Schumaker, Eds., Vanderbilt University Press, 89-96 (1998).

28. BIPM, IEC, IFCC, ILAC, ISO, IUPAC, IUPAP, and OIML, Evaluation of measurement data - Guide to the expression of uncertainty in measurement. Joint Committee for Guides in Metrology, JCGM 100:2008 (2008). http://www.bipm.org/utils/common/documents/jcgm/JCG M_100_2008_E.pdf.

29. BIPM, IEC, IFCC, ILAC, ISO, IUPAC, IUPAP, and OIML, Evaluation of measurement data - Supplement 1 to the "Guide to the expression of uncertainty in measurement" - Propagation of distributions using a Monte Carlo method. Joint Committee for Guides in Metrology, JCGM 101:2008 (2008). http://www.bipm.org/utils/common/documents/jcgm/JCG M_101_2008_E.pdf.

30. BIPM, IEC, IFCC, ILAC, ISO, IUPAC, IUPAP, and OIML, Evaluation of measurement data - Supplement 2 to the "Guide to the expression of uncertainty in measurement" - Extension to any number of output quantities. Joint Committee for Guides in Metrology, JCGM 102:2011 (2011).

http://www.bipm.org/utils/common/documents/jcgm/JCG M_102_2011_E.pdf

31. J. Wang, T. Li, "Iterative image reconstruction for CBCT using edge-preserving prior", Med Phys. 36: 252260 (2009). 